Article

\title{
On $r$-Central Incomplete and Complete Bell Polynomials
}

\author{
Dae San Kim ${ }^{1}{ }^{(\mathbb{D})}$, Han Young Kim ${ }^{2}$, Dojin Kim ${ }^{3, *}$ and Taekyun Kim ${ }^{2}$ \\ 1 Department of Mathematics, Sogang University, Seoul 04107, Korea; dskim@sogang.ac.kr \\ 2 Department of Mathematics, Kwangwoon University, Seoul 01897, Korea; gksdud213@kw.ac.kr (H.Y.K.); \\ tkkim@kw.ac.kr (T.K.) \\ 3 Department of Mathematics, Pusan National University, Busan 46241, Korea \\ * Correspondence: kimdojin@pusan.ac.kr
}

Received: 16 April 2019; Accepted:23 May 2019; Published: 27 May 2019

Abstract: Here we would like to introduce the extended $r$-central incomplete and complete Bell polynomials, as multivariate versions of the recently studied extended $r$-central factorial numbers of the second kind and the extended $r$-central Bell polynomials, and also as multivariate versions of the $r$ Stirling numbers of the second kind and the extended $r$-Bell polynomials. In this paper, we study several properties, some identities and various explicit formulas about these polynomials and their connections as well.

Keywords: extended $r$-central complete bell polynomials; extended $r$-central incomplete bell polynomials; complete $r$-Bell polynomials; incomplete $r$-bell polynomials

\section{Introduction}

We begin this section by briefly recalling several definitions related to the central factorial numbers of the second kind and the central Bell polynomials and also to their generalizations of the extended $r$-central factorial numbers of the second kind and the extended $r$-central Bell polynomials (see [1]). The central factorial $x^{[n]}$ is given by the generating function

$$
\left(\frac{t}{2}+\sqrt{1+\frac{t^{2}}{4}}\right)^{2 x}=\sum_{n=0}^{\infty} x^{[n]} \frac{t^{n}}{n !} .
$$

A proof of (1) can be found in [2], p. 215, Equations (27), (28) and (27a), (see also [1,3-5]).

It is well known that Formula (1) shows that

$$
x^{[0]}=1, \quad x^{[n]}=x\left(x+\frac{n}{2}-1\right) \cdots\left(x-\frac{n}{2}+1\right), \quad(n \geq 1),
$$

where $x^{[n]}$ is of degree $n$ in $x$.

The central factorial numbers of the second kind $T(n, k)$ are the coefficients in the expansion of $x^{n}$ in terms of central factorials as follows:

$$
x^{n}=\sum_{k=0}^{n} T(n, k) x^{[k]},
$$

(see [6-11]) and it is known that $T(2 n, 2 n-2 k)$ enumerates the number of ways to place $k$ rooks on a $3 \mathrm{D}$-triangle board of size $(n-1)$ (see $[12,13])$. The generating function of $T(n, k)$ is given by 


$$
\frac{1}{k !}\left(e^{\frac{t}{2}}-e^{-\frac{t}{2}}\right)^{k}=\sum_{n=k}^{\infty} T(n, k) \frac{t^{n}}{n !}
$$

which follows, for example, from (1) and (3).

Indeed, on the one hand by making use of (3) we have

$$
e^{x t}=\sum_{n=0}^{\infty} x^{n} \frac{t^{n}}{n !}=\sum_{k=0}^{\infty}\left(\sum_{n=k}^{\infty} T(n, k) \frac{t^{n}}{n !}\right) x^{[k]}
$$

On the other hand, by virtue of (1) we also have

$$
\begin{aligned}
e^{x t} & =\left(\frac{e^{\frac{t}{2}}-e^{-\frac{t}{2}}}{2}+\sqrt{1+\frac{\left(e^{\frac{t}{2}}-e^{-\frac{t}{2}}\right)}{4}}\right)^{2 x} \\
& =\sum_{k=0}^{\infty} \frac{1}{k !}\left(e^{\frac{t}{2}}-e^{-\frac{t}{2}}\right)^{k} x^{[k]} .
\end{aligned}
$$

Now, it can be easily seen that Equation (4) follows from (5) and (6).

Kim-Kim in [11] introduced the central Bell polynomials by means of generating function as

$$
e^{x\left(e^{\frac{t}{2}}-e^{-\frac{t}{2}}\right)}=\sum_{n=0}^{\infty} B_{n}^{(c)}(x) \frac{t^{n}}{n !} .
$$

We note by making use of (4) that identity (7) implies (see [1,11])

$$
B_{n}^{(c)}(x)=\sum_{k=0}^{n} T(n, k) x^{k}, \quad(n \geq 0) .
$$

For a nonnegative integer $r$, Kim-Dolgy-Kim-Kim in a recent work [1] introduced the extended $r$-central factorial numbers of the second kind given by the generating function:

$$
\frac{1}{k !}\left(e^{\frac{t}{2}}-e^{-\frac{t}{2}}\right)^{k} e^{r t}=\sum_{n=k}^{\infty} T^{(r)}(n+r, k+r) \frac{t^{n}}{n !} .
$$

From (8), it is noted that (see [1])

$$
(x+r)^{n}=\sum_{k=0}^{n} T^{(r)}(n+r, k+r) x^{[k]} .
$$

The extended $r$-central Bell polynomials [1] are defined by

$$
e^{x\left(e^{\frac{t}{2}}-e^{-\frac{t}{2}}\right)} e^{r t}=\sum_{n=0}^{\infty} B_{n}^{(c, r)}(x) \frac{t^{n}}{n !},
$$

By definition (10), it is also known that (see [1])

$$
B_{n}^{(c, r)}(x)=\sum_{k=0}^{n} x^{k} T^{(r)}(n+r, k+r), \quad(n \geq 0) .
$$


The purpose of this paper is to introduce and study the extended $r$-central incomplete and complete Bell polynomials, as multivariate versions of the recently studied the extended $r$-central factorial numbers of the second and the extended $r$-central Bell polynomials (see [1]), and also as multivariate versions of the $r$-Stirling numbers of the second kind and the extended $r$-Bell polynomials (see Section 2). Then we investigate their properties, some identities and various explicit formulas related to these polynomials and also their connections.

This paper is organized as follows. In Section 2, we introduce the incomplete and complete $r$-Bell polynomials and give some of their simple properties. We observe that these polynomials are multivariate versions of the $r$-Stirling numbers of the second kind and the extended $r$-Bell polynomials . In Section 3 , we introduce our object of study, namely the extended $r$-central incomplete and complete Bell polynomials, and provide several properties, some identities and various explicit formulas for them. Finally, in Section 4 , brief summaries for the obtained results about newly defined polynomials are provided.

\section{Preliminaries}

The $r$-Stirling numbers $S_{2}^{(r)}(n, k)$ of the second kind are defined by the generating function (see [14-19])

$$
\frac{1}{k !}\left(e^{t}-1\right)^{k} e^{r t}=\sum_{n=k}^{\infty} S_{2}^{(r)}(n+r, k+r) \frac{t^{n}}{n !}
$$

and they enumerate the number of partitions of the set $\{1,2, \cdots, n\}$ into $k$ nonempty disjoint subsets in such a way that $1,2, \cdots, r$ are in distinct subsets.

The extended $r$-Bell polynomials are given by (see [15])

$$
e^{r t} e^{x\left(e^{t}-1\right)}=\sum_{n=0}^{\infty} B_{n}^{(r)}(x) \frac{t^{n}}{n !} .
$$

One can show that Equations (12) and (13) imply

$$
\begin{aligned}
B_{n}^{(r)}(x) & =e^{-x} \sum_{k=0}^{\infty} \frac{(k+r)^{n}}{k !} x^{k} \\
& =\sum_{k=0}^{n} x^{k} S_{2}^{(r)}(n+r, k+r), \quad(n \geq 0) .
\end{aligned}
$$

In particular $x=1, B_{n}^{(c, r)}=B_{n}^{(c, r)}(1)$ are called the extended $r$-Bell numbers.

The incomplete $r$-Bell polynomials are given by the generating function

$$
\frac{1}{k !}\left(\sum_{j=1}^{\infty} x_{j} \frac{t^{j}}{j !}\right)^{k}\left(\sum_{j=0}^{\infty} y_{j+1} \frac{t^{j}}{j !}\right)^{r}=\sum_{n \geq k} B_{n+r, k+r}^{(r)}\left(x_{1}, x_{2}, \cdots ; y_{1}, y_{2} \cdots\right) \frac{t^{n}}{n !}
$$

Thus, we have

$$
\begin{aligned}
& B_{n+r, k+r}^{(r)}\left(x_{1}, x_{2}, \cdots ; y_{1}, y_{2} \cdots\right) \\
& \quad=\sum\left(\frac{n !}{k_{1} ! k_{2} ! \cdots}\left(\frac{x_{1}}{1 !}\right)^{k_{1}}\left(\frac{x_{2}}{2 !}\right)^{k_{2}} \cdots\right)\left(\frac{r !}{r_{0} ! r_{1} ! r_{2} ! \cdots}\left(\frac{y_{1}}{0 !}\right)^{r_{0}}\left(\frac{y_{2}}{1 !}\right)^{r_{1}}\left(\frac{y_{3}}{2 !}\right)^{r_{2}} \cdots\right),
\end{aligned}
$$

where the summation is over all integers $k_{1}, k_{2}, \cdots \geq 0$ and $r_{0}, r_{1}, r_{2} \cdots \geq 0$, such that 


$$
\sum_{i \geq 1} k_{i}=k, \quad \sum_{j \geq 0} r_{j}=r, \quad \text { and } \quad\left(k_{1}+r_{1}\right)+2\left(k_{2}+r_{2}\right)+3\left(k_{3}+r_{3}\right)+\cdots=n .
$$

Let $a_{1}, a_{2}, \cdots$, and $b_{1}, b_{2}, \cdots$ be any sequences of nonnegative integers. Then, as was noted in [20], $B_{n+r, k+r}^{(r)}\left(a_{1}, a_{2}, \cdots ; b_{1}, b_{2} \cdots\right)$ enumerates the number of partitions of a set with $(n+r)$ elements into $(k+r)$ blocks satisfying:

- $\quad$ The first $r$ elements are in different blocks,

- $\quad$ Any block of size $i$ with no elements of the first $r$ elements, can be colored with $a_{i}$ colors,

- $\quad$ Any block of size $i$ with one element of the first $r$ elements, can be colored with $b_{i}$ colors.

From (12) and (16), we note that

$$
\begin{gathered}
B_{n+r, k+r}^{(r)}(1,1, \cdots ; 1,1, \cdots)=S_{2}^{(r)}(n+k, k+r), \\
B_{n+r, k+r}^{(r)}\left(\alpha x_{1}, \alpha x_{2}, \cdots ; \alpha y_{1}, \alpha y_{2} \cdots\right)=\alpha^{k+r} B_{n+k, k+r}^{(r)}\left(x_{1}, x_{2}, \cdots ; y_{1}, y_{2} \cdots\right),
\end{gathered}
$$

and

$$
B_{n+r, k+r}^{(r)}\left(\alpha x_{1}, \alpha^{2} x_{2}, \cdots ; y_{1}, \alpha y_{2}, \alpha^{2} y_{3}, \cdots\right)=\alpha^{n} B_{n+k, k+r}^{(r)}\left(x_{1}, x_{2}, \cdots ; y_{1}, y_{2} \cdots\right),
$$

where $\alpha$ is a real number.

By using (15), we get

$$
\begin{aligned}
\sum_{n=k}^{\infty} B_{n+r, k+r}^{(r)}(x, 1,0,0, \cdots ; 1,0,0, \cdots) \frac{t^{n}}{n !} & =\frac{1}{k !}\left(x t+\frac{t^{2}}{2}\right)^{k} \\
& =t^{k} \frac{1}{k !} \sum_{n=0}^{k}\left(\begin{array}{c}
k \\
n
\end{array}\right)\left(\begin{array}{c}
t \\
2
\end{array}\right)^{n} x^{k-n} \\
& =\sum_{n=0}^{k} \frac{(n+k) !}{k !}\left(\begin{array}{l}
k \\
n
\end{array}\right)\left(\frac{1}{2}\right)^{n} x^{k-n} \frac{t^{n+k}}{(n+k) !} .
\end{aligned}
$$

Also, it can be seen that

$$
\sum_{n=k}^{\infty} B_{n+r, k+r}^{(r)}(x, 1,0,0, \cdots ; 1,0,0, \cdots) \frac{t^{n}}{n !}=\sum_{n=0}^{\infty} B_{n+k+r, k+r}^{(r)}(x, 1,0,0, \cdots ; 1,0,0, \cdots) \frac{t^{n+k}}{(n+k) !} .
$$

Thus, by (20) and (21), we have the following equation given by

$$
B_{n+k+r, k+r}^{(r)}(x, 1,0,0, \cdots ; 1,0,0, \cdots)= \begin{cases}\frac{(n+k) !}{k !}\left(\begin{array}{l}
k \\
n
\end{array}\right)\left(\frac{1}{2}\right)^{n} x^{k-n}, & \text { if } 0 \leq n \leq k, \\
0, & \text { if } n>k .\end{cases}
$$

By replacing $n$ by $n-k$ in (22), we get

$$
B_{n+r, k+r}^{(r)}(x, 1,0,0, \cdots ; 1,0,0, \cdots)=\frac{n !}{k !}\left(\begin{array}{c}
k \\
n-k
\end{array}\right) x^{2 k-n}\left(\frac{1}{2}\right)^{n-k}, \quad(k \leq n \leq 2 k) .
$$

Now, we define the complete $r$-Bell polynomials by virtue of generating function as 


$$
\exp \left(\sum_{i=1}^{\infty} x_{i} \frac{t^{i}}{i !}\right)\left(\sum_{j=0}^{\infty} y_{j+1} \frac{t^{j}}{j !}\right)^{r}=\sum_{n=0}^{\infty} B_{n}^{(r)}\left(x_{1}, x_{2}, \cdots ; y_{1}, y_{2}, \cdots\right) \frac{t^{n}}{n !} .
$$

From (15) and (24), we have

$$
\begin{aligned}
\sum_{n=0}^{\infty} B_{n}^{(r)}\left(x_{1}, x_{2}, \cdots ; y_{1}, y_{2}, \cdots\right) \frac{t^{n}}{n !} & =\sum_{k=0}^{\infty} \frac{1}{k !}\left(\sum_{i=1}^{\infty} x_{i} \frac{t^{i}}{i !}\right)^{k}\left(\sum_{j=0}^{\infty} y_{j+1} \frac{t^{j}}{j !}\right)^{r} \\
& =\sum_{k=0}^{\infty} \sum_{n=k}^{\infty} B_{n+r, k+r}^{(r)}\left(x_{1}, x_{2}, \cdots ; y_{1}, y_{2}, \cdots\right) \frac{t^{n}}{n !} \\
& =\sum_{n=0}^{\infty} \sum_{k=0}^{n} B_{n+r, k+r}^{(r)}\left(x_{1}, x_{2}, \cdots ; y_{1}, y_{2}, \cdots\right) \frac{t^{n}}{n !} .
\end{aligned}
$$

Comparing both sides of (25) gives us the identity

$$
B_{n}^{(r)}\left(x_{1}, x_{2}, \cdots ; y_{1}, y_{2}, \cdots\right)=\sum_{k=0}^{n} B_{n+r, k+r}^{(r)}\left(x_{1}, x_{2}, \cdots ; y_{1}, y_{2}, \cdots\right)
$$

Now, we observe that

$$
\begin{aligned}
B_{n}^{(r)}(x, x, \cdots ; 1,1, \cdots) & =\sum_{k=0}^{n} B_{n+r, k+r}^{(r)}(x, x, \cdots ; 1,1, \cdots) \\
& =\sum_{k=0}^{n} x^{k} B_{n+r, k+r}^{(r)}(1,1, \cdots ; 1,1, \cdots) \\
& =\sum_{k=0}^{n} x^{k} S_{2}^{(r)}(n+r, k+r) \\
& =B_{n}^{(r)}(x), \quad(n \geq 0) .
\end{aligned}
$$

\section{An Extended $r$-Central Complete and Incomplete Bell Polynomials}

Recently, in [21], we initiated the study of central incomplete Bell polynomials $T_{n, k}\left(x_{1}, x_{2}, \cdots, x_{n-k+1}\right)$ and the central complete Bell polynomials $B_{n}^{(c)}\left(x \mid x_{1}, x_{2}, \cdots, x_{n}\right)$, respectively given by

$$
\frac{1}{k !}\left(\sum_{m=1}^{\infty} \frac{1}{2^{m}}\left(x_{m}-(-1)^{m} x_{m}\right) \frac{t^{m}}{m !}\right)^{k}=\sum_{n=k}^{\infty} T_{n, k}\left(x_{1}, x_{2}, \cdots, x_{n-k+1}\right) \frac{t^{n}}{n !}
$$

and

$$
\exp \left(x \sum_{i=1}^{\infty} \frac{1}{2^{i}}\left(x_{i}-(-1)^{i} x_{i}\right) \frac{t^{i}}{i !}\right)=\sum_{n=0}^{\infty} B_{n}^{(c)}\left(x \mid x_{1}, x_{2}, \cdots, x_{n}\right) \frac{t^{n}}{n !}
$$

and studied some properties and identities concerning these polynomials. It was observed, in particular, that the number of partitioning a set with $n$ elements into $k$ blocks with odd sizes is given by the number of monomials appearing in $T_{n, k}\left(x_{1}, 2 x_{2}, \cdots, 2^{n-k} x_{n-k+1}\right)$, and that the number of partitioning a set with $n$ elements into a certain $k$ blocks with odd sizes is the coefficient of the corresponding monomial appearing in $T_{n, k}\left(x_{1}, 2 x_{2}, \cdots, 2^{n-k} x_{n-k+1}\right)$.

Here we will consider ' $r$-extensions' of the central incomplete and complete Bell polynomials. In light of (15), we may define the extended $r$-central incomplete Bell polynomials by 


$$
\frac{1}{k !}\left(\sum_{m=1}^{\infty}\left(\frac{1}{2}\right)^{m}\left(x_{m}-(-1)^{m} x_{m}\right) \frac{t^{m}}{m !}\right)^{k}\left(\sum_{j=0}^{\infty} y_{j+1} \frac{t^{j}}{j !}\right)^{r}=\sum_{n=k}^{\infty} T_{n+r, k+r}^{(r)}\left(x_{1}, x_{2}, \cdots ; y_{1}, y_{2}, \cdots\right) \frac{t^{n}}{n !}
$$

for any $k \in \mathbb{N} \cup\{0\}$. Then, for $n, k \geq 0$ with $n \geq k$, by (28), one can check that

$$
\begin{aligned}
T_{n+r, k+r}^{(r)}\left(x_{1}, x_{2}, \cdots ; y_{1}, y_{2}, \cdots\right)= & \sum\left(\frac{n !}{k_{1} ! k_{3} ! k_{5} ! \cdots}\left(\frac{x_{1}}{1 !}\right)^{k_{1}}\left(\frac{x_{3}}{2^{2} 3 !}\right)^{k_{3}}\left(\frac{x_{5}}{2^{4} 5 !}\right)^{k_{5}} \cdots\right) \\
& \times\left(\frac{r !}{r_{0} ! r_{1} ! r_{2} ! \cdots}\left(\frac{y_{1}}{0 !}\right)^{r_{0}}\left(\frac{y_{2}}{1 !}\right)^{r_{1}}\left(\frac{y_{3}}{2 !}\right)^{r_{2}} \cdots\right),
\end{aligned}
$$

where the summation is over all integers $k_{1}, k_{3}, k_{5} \cdots \geq 0$ and $r_{0}, r_{1}, r_{2} \cdots \geq 0$, such that

$$
\sum_{i \geq 1} k_{2 i-1}=k, \sum_{i \geq 0} r_{i}=r, \text { and } \sum_{i \geq 1}(2 i-1) k_{2 i-1}+\sum_{i \geq 1} i r_{i}=n
$$

The extended $r$-central incomplete Bell polynomials have the following combinatorial interpretation. This can be seen from (29). Let $a_{1}, a_{2}, \cdots$, and $b_{1}, b_{2}, \cdots$ be any sequences of nonnegative integers. Then $T_{n+r, k+r}^{(r)}\left(a_{1}, 2 a_{2}, 2^{2} a_{3}, \cdots ; b_{1}, b_{2}, b_{3}, \cdots\right)$ enumerates the number of partitions of a set with $(n+r)$ elements into $k$ blocks of odd sizes and $r$ blocks of any sizes satisfying:

- The first $r$ elements are in different blocks,

- Any block of (odd) size $i$ with no elements of the first $r$ elements, can be colored with $a_{i}$ colors,

- $\quad$ Any block of size $i$ with one element of the first $r$ elements, can be colored with $b_{i}$ colors.

From (15), (16) and (29), we note that

$$
T_{n+r, k+r}^{(r)}\left(x_{1}, x_{2}, \cdots ; y_{1}, y_{2}, \cdots\right)=B_{n+r, k+r}^{(r)}\left(x_{1}, 0, \frac{x_{3}}{2^{2}}, 0, \cdots ; y_{1}, y_{2}, y_{3} \cdots\right) .
$$

Therefore, we obtain the following theorem.

Theorem 1. For $n, k \geq 0$, with $n \geq k$, we have

$$
T_{n+r, k+r}^{(r)}\left(x_{1}, x_{2}, \cdots ; y_{1}, y_{2}, \cdots\right)=B_{n+r, k+r}^{(r)}\left(x_{1}, 0, \frac{x_{3}}{2^{2}}, 0, \cdots ; y_{1}, y_{2}, y_{3} \cdots\right) .
$$

From (28), we have

$$
\begin{aligned}
\sum_{n=k}^{\infty} T_{n+r, k+r}^{(r)}(1,1, \cdots ; 1,1, \cdots) \frac{t^{n}}{n !} & =\frac{1}{k !}\left(\sum_{m=1}^{\infty}\left(\frac{1}{2}\right)^{m}\left(1-(-1)^{m}\right) \frac{t^{m}}{m !}\right)^{k}\left(\sum_{j=0}^{\infty} \frac{t^{j}}{j !}\right)^{r} \\
& =\frac{1}{k !}\left(e^{\frac{t}{2}}-e^{-\frac{t}{2}}\right)^{k} e^{r t} \\
& =\sum_{n=k}^{\infty} T^{(r)}(n+r, k+r) \frac{t^{n}}{n !} .
\end{aligned}
$$

Therefore, by (32), we obtain the following corollary. 
Corollary 1. For $n, k \geq 0$, with $n \geq k$, we have

$$
T_{n+r, k+r}^{(r)}(1,1, \cdots ; 1,1, \cdots)=T^{(r)}(n+r, k+r), \quad(r \in \mathbb{N} \cup\{0\}) .
$$

Let $n, k$ be nonnegative integers. Then, from (28), we get

$$
\begin{aligned}
\sum_{n=k}^{\infty} T_{n+r, k+r}^{(r)}\left(x, x^{2}, x^{3}, \ldots ; 1, x, x^{2}, \cdots\right) \frac{t^{n}}{n !} & =\frac{1}{k !}\left(x t+\frac{x^{3}}{2^{2}} \frac{t^{3}}{3 !}+\frac{x^{5}}{2^{4}} \frac{t^{5}}{5 !}+\cdots\right)^{k}\left(1+x t+\frac{x^{2}}{2} t^{2}+\cdots\right)^{r} \\
& =\frac{1}{k !}\left(e^{\frac{x t}{2}}-e^{-\frac{x t}{2}}\right)^{k} e^{r x t} \\
& =\frac{1}{k !} \sum_{l-0}^{k}\left(\begin{array}{c}
k \\
l
\end{array}\right)(-1)^{k-l} e^{\left(l+r-\frac{k}{2}\right) x t} \\
& =\sum_{n=0}^{\infty} \frac{x^{n}}{k !} \sum_{l=0}^{k}\left(\begin{array}{l}
k \\
l
\end{array}\right)(-1)^{k-l}\left(l+r-\frac{k}{2}\right)^{n} \frac{t^{n}}{n !}
\end{aligned}
$$

Therefore, comparing both sides of (33) yields the following theorem.

Theorem 2. For $n, k \geq 0$, we have

$$
\frac{x^{n}}{k !} \sum_{l=0}^{k}\left(\begin{array}{l}
k \\
l
\end{array}\right)(-1)^{k-l}\left(l+r-\frac{k}{2}\right)^{n}= \begin{cases}T_{n+r, k+r}^{(r)}\left(x, x^{2}, x^{3}, \ldots ; 1, x, x^{2}, \cdots\right), & \text { if } n \geq k \\
0, & \text { otherwise. }\end{cases}
$$

In [10], Kim-Dolgy-Kim-Kim proved the following equation (34) given by

$$
\frac{1}{k !} \sum_{l=0}^{k}\left(\begin{array}{l}
k \\
l
\end{array}\right)(-1)^{k-l}\left(l+r-\frac{k}{2}\right)^{n}= \begin{cases}T^{(r)}(n+r, k+r), & \text { if } n \geq k, \\
0, & \text { otherwise }\end{cases}
$$

where $n, k \in \mathbb{Z}$ with $n, k \geq 0$. Therefore, by (34), the following corollary is established.

Corollary 2. For $n, k \in \mathbb{N} \cup\{0\}$, with $n \geq k$, we have

$$
T_{n+r, k+r}^{(r)}\left(x, x^{2}, x^{3}, \ldots ; 1, x, x^{2}, \cdots\right)=x^{n} T^{(r)}(n+r, k+r) .
$$

From (29) and Corollary 2 , one can also have the following identity.

Corollary 3. For $n, k \geq 0$, with $n \geq k$, we have

$$
T_{n+r, k+r}^{(r)}\left(x, x^{2}, x^{3}, \ldots ; 1, x, x^{2}, \cdots\right)=x^{n} T_{n+r, k+r}^{(r)}(1,1, \cdots ; 1,1, \cdots)
$$


and

$$
\begin{aligned}
T_{n+r, k+r}^{(r)}(1,1, \cdots ; 1,1, \cdots)= & T^{(r)}(n+r, k+r) \\
= & B_{n+r, r}^{(r)}\left(1,0, \frac{1}{2^{2}}, 0, \frac{1}{2^{4}}, 0, \cdots ; 1,1,1, \cdots\right) \\
= & \sum\left(\frac{n !}{k_{1} ! k_{3} ! k_{5} ! \cdots}\left(\frac{1}{1 !}\right)^{k_{1}}\left(\frac{1}{2^{2} 3 !}\right)^{k_{3}}\left(\frac{1}{2^{4} 5 !}\right)^{k_{5}} \cdots\right) \\
& \times\left(\frac{r !}{r_{0} ! r_{1} ! r_{2} ! \cdots}\left(\frac{1}{0 !}\right)^{r_{0}}\left(\frac{1}{1 !}\right)^{r_{1}}\left(\frac{1}{2 !}\right)^{r_{2}} \cdots\right),
\end{aligned}
$$

where the summation is over all integers $k_{1}, k_{3}, k_{5} \cdots \geq 0$ and $r_{0}, r_{1}, r_{2} \cdots \geq 0$, satisfying the conditions in (30).

For $n, k \geq 0$, we have

$$
\sum_{n=k}^{\infty} T_{n+r, k+r}^{(r)}(x, 1,0,0, \cdots ; 1,0,0, \cdots) \frac{t^{n}}{n !}=\frac{1}{k !}(x t)^{k} .
$$

By comparing the coefficients on both sides of (35), we have

$$
T_{n+r, k+r}^{(r)}(x, 1,0,0, \cdots ; 1,0,0, \cdots)=x^{k}\left(\begin{array}{c}
0 \\
n-k
\end{array}\right) .
$$

Also, by (29), one can obtain that

$$
\begin{aligned}
T_{n+r, k+r}^{(r)}(x, x, \cdots ; y, y, \cdots) & =x^{k} y^{r} T_{n+r, k+r}^{(r)}(1,1, \cdots ; 1,1, \cdots) \\
& =x^{k} y^{r} T^{(r)}(n+r, k+r),
\end{aligned}
$$

and

$$
T_{n+r, k+r}^{(r)}\left(\alpha x_{1}, \alpha x_{2}, \cdots ; \alpha y_{1}, \alpha y_{2}, \cdots\right)=\alpha^{k+r} T_{n+r, k+r}^{(r)}\left(x_{1}, x_{2}, \cdots ; y_{1}, y_{2}, \cdots\right),
$$

where $n, k$ are nonnegative integers with $n \geq k$.

Now, we observe that

$$
\begin{aligned}
\exp \left(x \sum_{i=1}^{\infty}\left(\frac{1}{2}\right)^{i}\left(x_{i}-(-1)^{i} x_{i}\right) \frac{t^{i}}{i !}\right) & \left(\sum_{j=0}^{\infty} y_{j+1} \frac{t^{j}}{j !}\right)^{r} \\
& =\sum_{k=0}^{\infty} x^{k} \frac{1}{k !}\left(\sum_{i=1}^{\infty}\left(\frac{1}{2}\right)^{i}\left(x_{i}-(-1)^{i} x_{i}\right) \frac{t^{i}}{i !}\right)^{k}\left(\sum_{j=0}^{\infty} y_{j+1} \frac{t^{j}}{j !}\right)^{r} \\
& =\sum_{k=0}^{\infty} x^{k} \sum_{n=k}^{\infty} T_{n+r, k+r}^{(r)}\left(x_{1}, x_{2}, \cdots ; y_{1}, y_{2}, \cdots\right) \frac{t^{n}}{n !} \\
& =\sum_{k=0}^{\infty} \sum_{k=0}^{n} x^{k} T_{n+r, k+r}^{(r)}\left(x_{1}, x_{2}, \cdots ; y_{1}, y_{2}, \cdots\right) \frac{t^{n}}{n !}
\end{aligned}
$$

Taking (24) into account, we may define the extended $r$-central complete Bell polynomials by

$$
\exp \left(x \sum_{i=1}^{\infty}\left(\frac{1}{2}\right)^{i}\left(x_{i}-(-1)^{i} x_{i}\right) \frac{t^{i}}{i !}\right)\left(\sum_{j=0}^{\infty} y_{j+1} \frac{t^{j}}{j !}\right)^{r}=\sum_{k=0}^{\infty} B_{n}^{(c, r)}\left(x \mid x_{1}, x_{2}, \cdots ; y_{1}, y_{2}, \cdots\right) \frac{t^{n}}{n !}
$$


In particular, when $x=1, B_{n}^{(c, r)}\left(1 \mid x_{1}, x_{2}, \cdots ; y_{1}, y_{2}, \cdots\right)=B_{n}^{(c, r)}\left(x_{1}, x_{2}, \cdots ; y_{1}, y_{2}, \cdots\right)$ are called the extended $r$-central complete Bell numbers.

For $n \geq 0$, by (39) and (40), we get

$$
B_{n}^{(c, r)}\left(x_{1}, x_{2}, \cdots ; y_{1}, y_{2}, \cdots\right)=\sum_{k=0}^{n} T_{n+r, k+r}^{(r)}\left(x_{1}, x_{2}, \cdots ; y_{1}, y_{2}, \cdots\right)
$$

and

$$
B_{n}^{(c, r)}\left(x \mid x_{1}, x_{2}, \cdots ; y_{1}, y_{2}, \cdots\right)=\sum_{k=0}^{n} x^{k} T_{n+r, k+r}^{(r)}\left(x_{1}, x_{2}, \cdots ; y_{1}, y_{2}, \cdots\right)
$$

It is easily noted that $B_{0}^{(c, r)}\left(x_{1}, x_{2}, \cdots ; y_{1}, y_{2}, \cdots\right)=y_{1}^{r}$.

Hence, one can have the following theorem.

Theorem 3. For $n \geq 0$, we have

$$
B_{n}^{(c, r)}\left(x \mid x_{1}, x_{2}, \cdots ; y_{1}, y_{2}, \cdots\right)=\sum_{k=0}^{n} x^{k} T_{n+r, k+r}^{(r)}\left(x_{1}, x_{2}, \cdots ; y_{1}, y_{2}, \cdots\right)
$$

and

$$
B_{n}^{(c, r)}\left(x_{1}, x_{2}, \cdots ; y_{1}, y_{2}, \cdots\right)=\sum_{k=0}^{n} T_{n+r, k+r}^{(r)}\left(x_{1}, x_{2}, \cdots ; y_{1}, y_{2}, \cdots\right) .
$$

Please note that

$$
\begin{aligned}
B_{n}^{(c, r)}(1,1, \cdots ; 1,1, \cdots) & =\sum_{k=0}^{n} T_{n+r, k+r}^{(r)}(1,1, \cdots ; 1,1, \cdots) \\
& =\sum_{k=0}^{n} T^{(r)}(n+r, k+r) \\
& =B_{n}^{(c, r)},
\end{aligned}
$$

and

$$
B_{n}^{(c, r)}(x \mid 1,1, \cdots ; 1,1, \cdots)=\sum_{k=0}^{n} x^{k} T^{(r)}(n+r, k+r)=B_{n}^{(c, r)}(x), \quad(n \geq 0) .
$$

By (39), we get

$$
\begin{aligned}
\exp & \left(\sum_{i=1}^{\infty}\left(\frac{1}{2}\right)^{i}\left(x_{i}-(-1)^{i} x_{i}\right) \frac{t^{i}}{i !}\right)\left(\sum_{j=0}^{\infty} y_{j+1} \frac{t^{j}}{j !}\right)^{r} \\
= & \sum_{n=0}^{\infty}\left\{\sum\left(\frac{n !}{k_{1} ! k_{3} ! k_{5} ! \cdots}\left(\frac{x_{1}}{1 !}\right)^{k_{1}}\left(\frac{x_{3}}{2^{2} 3 !}\right)^{k_{3}}\left(\frac{x_{5}}{2^{4} 5 !}\right)^{k_{5}} \cdots\right)\right. \\
& \left.\times\left(\frac{r !}{r_{0} ! r_{1} ! r_{2} ! \cdots}\left(\frac{y_{1}}{0 !}\right)^{r_{0}}\left(\frac{y_{2}}{1 !}\right)^{r_{1}}\left(\frac{y_{3}}{2 !}\right)^{r_{2}} \cdots\right)\right\} \frac{t^{n}}{n !},
\end{aligned}
$$

where the inner sum runs over all integers $k_{1}, k_{3}, k_{5} \cdots \geq 0$ and $r_{0}, r_{1}, r_{2} \cdots \geq 0$, such that

$$
\sum_{i \geq 0} r_{i}=r, \text { and } \sum_{i \geq 1}(2 i-1) k_{2 i-1}+\sum_{i \geq 1} i r_{i}=n
$$


For $n \geq 0$, we have

$$
\begin{aligned}
B_{n}^{(c, r)}\left(x_{1}, x_{2}, \cdots ; y_{1}, y_{2}, \cdots\right)= & \sum_{k=0}^{n} T_{n+r, k+r}^{(r)}\left(x_{1}, x_{2}, \cdots ; y_{1}, y_{2}, \cdots\right) \\
= & \sum\left(\frac{n !}{k_{1} ! k_{3} ! k_{5} ! \cdots}\left(\frac{x_{1}}{1 !}\right)^{k_{1}}\left(\frac{x_{3}}{2^{2} 3 !}\right)^{k_{3}}\left(\frac{x_{5}}{2^{4} 5 !}\right)^{k_{5}} \cdots\right) \\
& \times\left(\frac{r !}{r_{0} ! r_{1} ! r_{2} ! \cdots}\left(\frac{y_{1}}{0 !}\right)^{r_{0}}\left(\frac{y_{2}}{1 !}\right)^{r_{1}}\left(\frac{y_{3}}{2 !}\right)^{r_{2}} \cdots\right),
\end{aligned}
$$

where the sum is over all integers $k_{1}, k_{3}, k_{5} \cdots \geq 0$ and $r_{0}, r_{1}, r_{2} \cdots \geq 0$, satisfying the conditions in (43).

Thus, the following theorem is established.

Theorem 4. For $n \geq 0$, we have

$$
\begin{aligned}
B_{n}^{(c, r)}\left(x_{1}, x_{2}, \cdots ; y_{1}, y_{2}, \cdots\right)= & \sum\left(\frac{n !}{k_{1} ! k_{3} ! k_{5} ! \cdots}\left(\frac{x_{1}}{1 !}\right)^{k_{1}}\left(\frac{x_{3}}{2^{2} 3 !}\right)^{k_{3}}\left(\frac{x_{5}}{2^{4} 5 !}\right)^{k_{5}} \cdots\right) \\
& \times\left(\frac{r !}{r_{0} ! r_{1} ! r_{2} ! \cdots}\left(\frac{y_{1}}{0 !}\right)^{r_{0}}\left(\frac{y_{2}}{1 !}\right)^{r_{1}}\left(\frac{y_{3}}{2 !}\right)^{r_{2}} \cdots\right),
\end{aligned}
$$

where the sum is over all integers $k_{1}, k_{3}, k_{5} \cdots \geq 0$ and $r_{0}, r_{1}, r_{2} \cdots \geq 0$, satisfying the conditions in (43).

Now, we observe that

$$
\begin{aligned}
\exp \left(x \sum_{i=1}^{\infty}\left(\frac{1}{2}\right)^{i}\left(1-(-1)^{i}\right) \frac{t^{i}}{i !}\right)\left(\sum_{j=0}^{\infty} \frac{t^{j}}{j !}\right)^{r} & =\sum_{k=0}^{\infty} x^{k} \frac{1}{k !}\left(\sum_{i=1}^{\infty}\left(\frac{1}{2}\right)^{i}\left(1-(-1)^{i}\right) \frac{t^{i}}{i !}\right)^{k}\left(\sum_{j=0}^{\infty} \frac{t^{j}}{j !}\right)^{r} \\
& =\sum_{k=0}^{\infty} x^{k} \sum_{n=k}^{\infty} T_{n+r, k+r}^{(r)}(1,1, \cdots ; 1,1, \cdots) \frac{t^{n}}{n !} \\
& =\sum_{n=0}^{\infty} \sum_{k=0}^{n} x^{k} T_{n+r, k+r}^{(r)}(1,1, \cdots ; 1,1, \cdots) \frac{t^{n}}{n !}
\end{aligned}
$$

Alternatively, the left hand side of (45) can be simplified in the following way:

$$
\exp \left(x \sum_{i=1}^{\infty}\left(\frac{1}{2}\right)^{i}\left(1-(-1)^{i}\right) \frac{t^{i}}{i !}\right)\left(\sum_{j=0}^{\infty} \frac{t^{j}}{j !}\right)^{r}=e^{x\left(e^{\frac{t}{2}}-e^{-\frac{t}{2}}\right)} e^{r t}=\sum_{n=0}^{\infty} B_{n}^{(c, r)}(x) \frac{t^{n}}{n !} .
$$

Comparing the coefficients in (45) and (46) gives the following identity.

Theorem 5. For $n \geq 0$, we have

$$
\sum_{k=0}^{n} x^{k} T_{n+r, k+r}^{(r)}(1,1, \cdots ; 1,1, \cdots)=B_{n}^{(c, r)}(x) .
$$

From (29), it is noted that

$$
\begin{aligned}
\sum_{k=0}^{n} x^{k+r} T_{n+r, k+r}^{(r)}(1,1, \cdots ; 1,1, \cdots) & =\sum_{k=0}^{n} T_{n+r, k+r}^{(r)}(x, x, \cdots ; x, x, \cdots) \\
& =B_{n}^{(c, r)}(x, x, \cdots ; x, x, \cdots)
\end{aligned}
$$

which yields the next corollary. 
Corollary 4. For $n \geq 0$, we have

$$
B_{n}^{(c, r)}(x, x, \cdots ; x, x, \cdots)=x^{r} B_{n}^{(c, r)}(x) .
$$

\section{Conclusions}

In recent years, studies on various old and new special numbers and polynomials have received attention from many mathematicians. They have been carried out by several means, including generating functions, combinatorial methods, umbral calculus, $p$-adic analysis, differential equations, probability and so on.

In this paper, by making use of generating functions we introduced and studied the extended $r$-central incomplete and complete Bell polynomials, as multivariate versions of the recently studied the extended $r$-central factorial numbers of the second and the extended $r$-central Bell polynomials (see [1]), and also as multivariate versions of the $r$-Stirling numbers of the second kind and the extended $r$-Bell polynomials (see Section 2). Then we studied several properties, some identities and various explicit formulas related to these polynomials and also their connections.

In Section 1 we briefly recalled, in more detail, definitions and basic properties about the central factorial numbers of the second kind, the central Bell polynomials, the extended $r$-central factorial numbers of the second kind and the extended $r$-central Bell polynomials. In Section 2 we introduced the incomplete and complete $r$-Bell polynomials as multivariate versions of the $r$-Stirling numbers of the second kind and the extended $r$-Bell polynomials and give some of their simple properties. In Section 3, we introduced the extended $r$-central incomplete and complete Bell polynomials, and provided several properties, some identities and various explicit formulas for them.

As our immediate next project, we would like to find some further results about the extended $r$-central incomplete and complete Bell polynomials by virtue of umbral calculus and also some of their applications associated with partition polynomials.

Author Contributions: Conceptualization, D.S.K. and T.K.; Formal analysis, D.S.K., D.K. and T.K.; Funding acquisition, D.K.; Investigation, D.S.K., H.Y.K. and T.K.; Methodology, D.S.K. and T.K.; Project administration, T.K.; Software, D.K.; Supervision, D.S.K. and T.K.; Validation, D.S.K., H.Y.K., D.K. and T.K.; Visualization, H.Y.K. and D.K.; Writing一original draft, T.K.; Writing - review \& editing, D.S.K., H.Y.K. and D.K.

Funding: This work was supported by the National Research Foundation of Korea (NRF) grant funded by the Korea government (MSIT) (No. 2019R1C1C1003869).

Conflicts of Interest: The authors declare no conflict of interest.

Acknowledgments: We would like to thank the referees for their comments and suggestions which improved the original manuscript greatly.

\section{References}

1. Kim, D.S.; Dolgy, D.V.; Kim, D.; Kim, T. Some identities on $r$-central factorial numbers and $r$-central Bell polynomials. arXiv 2019, arXiv:1903.11689v1.

2. Riordan, J. Combinatorial Identities; John Wiley \& Sons, Inc.: New York, NY, USA, 1968.

3. Kim, T.; Kim, D.S.; Jang, G.-W.; Kwon, J. Extended central factorial polynomials of the second kind. Adv. Differ. Equ. 2019, 24. [CrossRef]

4. Kim, T.; Kim, D.S. Degenerate central Bell numbers and polynomials. Rev. Real Acad. Clenc. Exactas Fis. Nat. Ser. A Mat. 2019, 1-7. [CrossRef]

5. Zhang, W. Some identities involving the Euler and the central factorinal numbers. Fibonacci Quart. 1998, 36, 154-157.

6. Butzer, P.L.; Schmidt, M.; Stark, E.L.; Vogt, L. Central factorial numbers; their main properties and some applications. Numer. Funct. Anal. Optim. 1989, 10, 419-488. [CrossRef] 
7. Carlitz, L.; Riordan, J. The divided central differences of zero. Canad. J. Math. 1963, 15, 94-100. [CrossRef]

8. Carlitz, L. Some remarks on the Bell numbers. Fibonacci Quart. 1980 , 18, 66-73.

9. Charalambides, C.A. Central factorial numbers and related expansions. Fibonacci Quart. 1981, 19, 451-456.

10. Kim, T. A note on central factorial numbers. Proc. Jangjeon Math. Soc. 2018, 21, 575-588.

11. Kim, T.; Kim, D.S. A note on central Bell numbers and polynomials. Adv. Stud. Contemp. Math. (Kyungshang) 2019, 27, 289-298.

12. Belbachir, H.; Djemmada, Y. On central Fubini-like numbers and polynomials. arXiv 2018, arXiv:1811.06734v1.

13. Krzywonos, N.; Alayont, F. Rook polynomials in higher dimensions. Stud. Summer Sch. 2009, 29; Available online: https: / / scholarworks.gvsu.edu/sss/29/ (accessed on 2 March 2019).

14. Duran, U.; Acikgoz, M.; Araci, S. On $(q, r, w)$-Stirling numbers of the second kind. J. Inequal. Spec. Funct. 2018, 9, 9-16.

15. Kim, T.; Yao, Y.; Kim, D.S.; Jang, G.-W. Degenerate $r$-Stirling numbers and $r$-Bell polynomials. Russ. J. Math. Phys. 2018, 25, 44-58. [CrossRef]

16. Pyo, S.-S. Degenerate Cauchy numbers and polynomials of the fourth kind. Adv. Stud. Contemp. Math. (Kyungshang) 2018, 28, 127-138.

17. Roman, S. The umbral calculus. In Pure and Applied Mathematics; Harcourt Brace Jovanovich: New York, NY, USA, 1984.

18. Simsek, Y. Identities and relations related to combinatorial numbers and polynomials. Proc. Jangjeon Math. Soc. 2017, 20, 127-135.

19. Simsek, Y. Identities on the Changhee numbers and Apostol-type Daehee polynomials. Adv. Stud. Contemp. Math. (Kyungshang) 2017, 27, 199-212.

20. Mihoubi, M.; Rahmani, M. The partial r-Bell polynomials. Afr. Mat. 2017, 28, 1167-1183. [CrossRef]

21. Kim, T.; Kim, D.S.; Jang, G.-W. On central complete and incomplete Bell polynomials I. Symmetry 2019, 11, 288. [CrossRef]

(C) 2019 by the authors. Licensee MDPI, Basel, Switzerland. This article is an open access article distributed under the terms and conditions of the Creative Commons Attribution (CC BY) license (http:/ / creativecommons.org/licenses/by/4.0/). 